\title{
News
}

Forsch Komplementmed 23 | 6 | 16

\section{Vitamin D und Magnesium}

\section{Synergien nutzen - gemeinsam substituieren}

Die Versorgung mit Vitamin D ist bei einem Großteil der Bevölkerung unzureichend. Ein Vitamin-D-Defizit schwächt die Knochen und erhöht das Risiko für Herz-KreislaufErkrankungen. Durch eine frühzeitige Substitution ließen sich weitreichende Folgeschäden vermeiden. Wenig bekannt ist, dass eine Kombination von Vitamin D und Magnesium besonders wirksam zu sein scheint. Wie die beiden Nährstoffe im Körper zusammenspielen und sich diese Synergie nutzen lässt, berichteten Experten auf dem Vitamin-DSymposium im Rahmen der Medizinischen Woche in Baden-Baden.

Mehr als die Hälfte der deutschen Bevölkerung ist nicht ausreichend mit Vitamin D versorgt (Serumkonzentration $<20 \mathrm{ng} / \mathrm{ml}$ ) [1] Dabei ist die Vitamin-D-Unterversorgung nicht nur ein nationales Problem, wie Apotheker Uwe Gröber (Essen) auf dem Fachsymposium mitteilte. Eine aktuelle Auswertung von 14 Studien mit knapp 56000 Personen aus Europa zeige, dass 40\% der Teilnehmer eine Vitamin-D-Serumkonzentration von unter $20 \mathrm{ng} / \mathrm{ml}$ und somit eine VitaminD-Insuffizienz aufweisen [2].

Das Nährstoffdefizit $\left(25(\mathrm{OH}) \mathrm{D}_{3}<20 \mathrm{ng} / \mathrm{ml}\right)$ gelte als wichtiger ätiologischer Faktor bei der Pathogenese verschiedener chronischer Erkrankungen wie Knochen- und Herz-Kreislauf-Erkrankungen sowie Diabetes mellitus.

Interaktionen von Vitamin D und Magnesium

Was häufig nicht bedacht wird: Auch eine unzureichende Magnesiumversorgung begünstigt die Entstehung eines Vitamin-D-
Defizits. Wie Professor Stefan Pilz (Graz) berichtete, wird Magnesium benötigt, um Vitamin D3 in seine aktive Form, das 1,25Dihydroxy-Vitamin D, umzuwandeln. Vitamin D fördert wiederum die Aufnahme von Magnesium im Darm.

Der Mineralstoff dient als Kofaktor bei über 600 enzymatischen Reaktionen und ist vor allem für das Herz-Kreislauf-System, aber auch für den Glukose- und Knochenstoffwechsel von Bedeutung [3]. Pilz wies darauf hin, dass sich eine Rachitis nur effektiv durch eine Vitamin-D-Supplementation behandeln lässt, wenn kein Magnesiummangel vorliegt bzw. dieser ebenfalls ausgeglichen wird.

Besteht bei einer Unterversorgung mit Vitamin D gleichzeitig eine geringe Magnesiumzufuhr, erhöht sich auch das Gesamtmortalitätsrisiko (Abb. 1). Im Vergleich zu den Studienteilnehmern mit den höchsten VitaminD-Konzentrationen hatten Probanden mit den geringsten Werten ein um 31\% höheres Sterblichkeitsrisiko. Lag gleichzeitig eine zu geringe Magnesiumaufnahme vor, erhöhte sich das Risiko zusätzlich um 29\% und lag signifikant höher im Vergleich zu den Personen ohne Unterversorgung [4]. Insbesondere bei Patienten mit Herz-Kreislauf-Erkrankungen, Diabetes mellitus oder arterieller Hypertonie liege jedoch vermehrt ein Defizit beider Nährstoffe vor, bemerkte Pilz.

\section{Kombinierte Supplementation - je nach Bedarf}

Die Osteoporose sei eine wegen des demografischen Wandels zunehmende, aber weiterhin unterschätzte Erkrankung, betonte

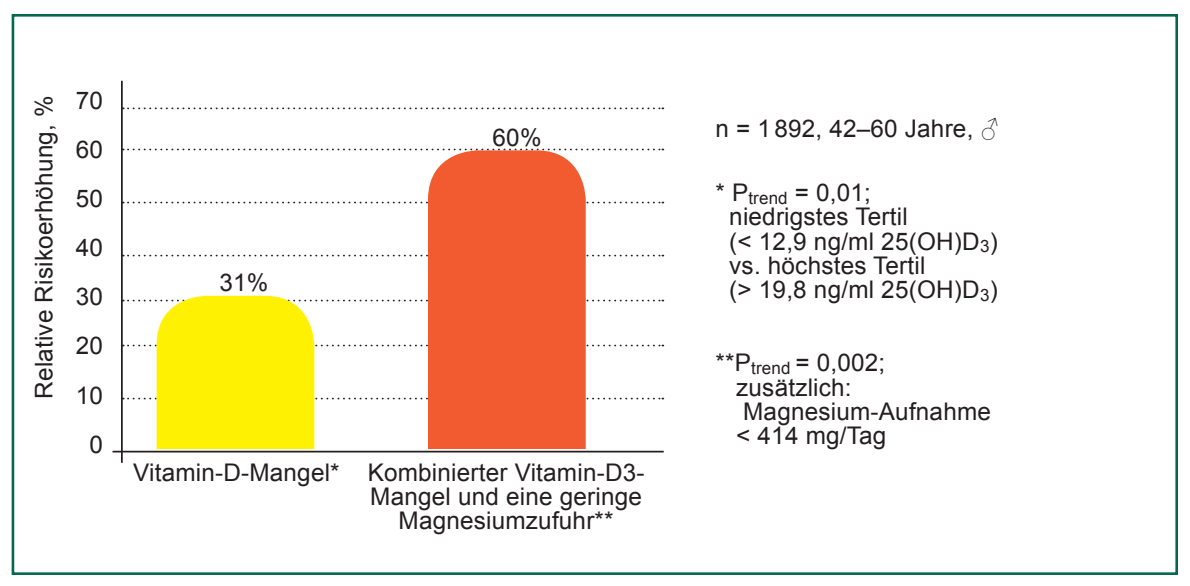

Abb. 1. Zusammenhang zwischen Mortalitätsrisiko und Vitamin-D-Mangel bzw. kombiniertem VitaminD-Mangel und geringer Magnesiumzufuhr (nach Mursu et al., 2015).

\section{Referenzbereich Vitamin D (nach Gröber)}

$<20 \mathrm{ng} / \mathrm{ml}(<50 \mathrm{nmol} / \mathrm{l})$ :

Mangel

$<30 \mathrm{ng} / \mathrm{ml}(<75 \mathrm{nmol} / \mathrm{l})$ :

Insuffizienz, moderater Mangel

$30-100 \mathrm{ng} / \mathrm{ml}(\mathbf{7 5 - 2 5 0} \mathrm{nmol} / \mathrm{l})$ :

adäquate Versorgung

$40-60 \mathrm{~g} / \mathrm{ml}(100-150 \mathrm{nmol} / \mathrm{l})$ :

optimale Versorgung

$>100 \mathrm{ng} / \mathrm{ml}(>250 \mathrm{nmol} / \mathrm{l})$ :

zu hoch

Referenzbereich Magnesium

$0,76 \mathrm{mmol} / \mathrm{l}$ :

Hypomagnesiämie

0,76-1,1 mmol/1:

adäquate Versorgung

Professor Franz Jakob (Würzburg). Zu den modifizierbaren Risikofaktoren der Osteoporose gehöre neben einer Vitamin-D- und Calcium- auch eine Magnesiumunterversorgung. Ein Calciumdefizit kann in den meisten Fällen alimentär ausgeglichen werden. Es empfiehlt sich daher, sowohl den VitaminD- als auch den Magnesiumstatus im Blick zu haben und bei Bedarf beide Nährstoffe gemeinsam zu substituieren. Diese Kombination bietet das neue Nahrungsergänzungsmittel Vitagamma ${ }^{\circledR}$ D3 Duo mit 1000 I.E. Vitamin D3 und $150 \mathrm{mg}$ Magnesium pro Tablette.

\section{Quelle}

Vitamin-D-Symposium der Wörwag Pharma GmbH: Vitamin D und Magnesium - Gemeinsam sind sie stark! Medizinische Woche, Baden-Baden, 1. November 2016.

\section{Literatur}

1 Hintzpeter B et al.: Eur J Clin Nutr 2008;62:10791089.

2 Cashman KD et al.: Am J Clin Nutr 2016;103:10331044.

3 Gröber U et al.: Nutrients 2015;7:8199-8226.

4 Mursu J et al.: Eur J Epidemiol 2015;30:343-347.

Weitere Informationen bei

Wörwag Pharma GmbH \& Co. KG

Calwer Str. 7

71034 Böblingen

info@woerwagpharma.de

www.woerwagpharma.de 


\section{News}

Forsch Komplementmed 23 | 6 | 16

Wissenschaftliche Beiträge des 6. Mistelsymposiums publiziert

\section{Die Mistel in der Tumortherapie 4 - Aktueller Stand der Forschung und klinische Anwendung}

Seit 1995 finden alle 4 Jahre die international und interdisziplinär ausgerichteten Symposien zum Thema «Die Mistel in der Tumortherapie» statt. Die Mistelsymposien sind fest in Medizin und Pharmazie verankert. Im November 2015 trafen sich zum 6. Mal Wissenschaftler, Ärzte und andere in den Gesundheitsberufen Tätige, um wissenschaftliche Beiträge aus der Biologie, der Pharmazie und Pharmakologie, der Klinik und Präklinik, Erkenntnisse aus therapeutischer Erfahrung sowie klinische Studien zu präsentieren. Wie vor 4 Jahren wurde auch bei dieser Tagung eine Tumorentität genauer betrachtet: Gastrointestinale Tumoren. Das Buch «Die Mistel in der Tumortherapie 4 - Aktueller Stand der Forschung und klinische Anwendung» doku- mentiert die Ergebnisse des Symposiums und damit den aktuellen Stand der Forschung und der klinischen Anwendung. Forschung auf dem Gebiet der Onkologie kann nur interdisziplinär fruchtbar sein, um mit den gewonnenen Ergebnissen den hohen Erwartungen der Hilfe und Rat suchenden Patienten zu entsprechen.

Weitere Informationen bei Carl Gustav Carus-Institut Dr. Rainer Scheer

Am Eichhof 30

75223 Niefern-Öschelbronn

info@carus-institut.de

www.carus-institut.de
Autoren

Rainer Scheer

Susanne Alban

Hans Becker

André-Michael Beer

Wolfgang Blaschek

Wolfgang Kreis

Harald Matthes

Heinz Schilcher $t$

Günther Spahn

Rainer Stange (Hrsg.)

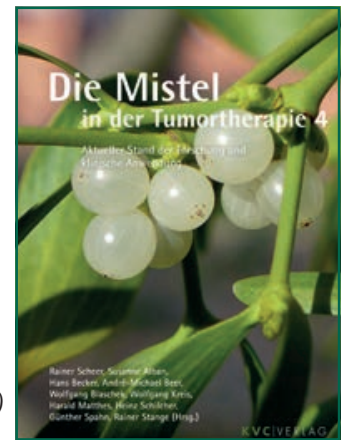

Essen 2016; 469 Seiten; ISBN 978-3-945150-68-9

$\dagger$ Heinz Schilcher starb am 17. Juni 2015. Die Mitorganisatoren des Symposiums sind überaus dankbar für seinen langjährigen und zuverlässigen Einsatz und seine vielfältigen Beiträge zu den Mistelsymposien.

\section{Stressbewältigung}

\section{Innere Stärke gewinnen}

Die niedergelassenen Ärzte sehen sich zunehmend Patienten gegenüber, die unter stressbedingten Symptomen wie Herzrasen, Schlafstörungen, Reizdarm usw leiden, und sowohl Diagnostik als auch Behandlung erweisen sich häufig als schwierig. «Wenn eine organische Erkrankung ausgeschlossen ist, hat der Arzt zwar einen wichtigen Teil seiner Arbeit getan, dem Patienten geht es aber immer noch schlecht», betont Dr. Tobias Sprenger, Facharzt für Allgemeinmedizin mit dem Schwerpunkt Anthroposophische Medizin aus Köln. «Die häufig verschriebenen Beruhigungsmittel, Schlaftabletten, Betablocker usw können zwar vorübergehend die Symptome unterdrücken, lösen aber häufig das Problem nicht und können Nebenwirkungen haben, die den Patienten belas- ten.» Gerade bei den Fehlregulationen des Organismus bei Stress ist die Anthroposophische Medizin ein multimodaler Ansatz, der medikamentöse wie auch eine breite Palette von nichtmedikamentösen Maßnahmen beinhaltet. Das Ziel ist zum einen, die physischen wie psychischen Stressfolgen zu lindern, zum anderen, die körperliche Fitness, die mentale Stärke und auch eine realistische Selbstwahrnehmung wiederherzustellen und zu festigen. Eine empfehlenswerte Übung zur Achtsamkeit sei das «Mindful Walking», so Dr. Sprenger, das die Wahrnehmung des eigenen Zustands, der eigenen Bedürfnisse und Grenzen sowie der Umwelt stärkte. Auch medikamentös kann man den Reaktionen des Organismus auf die Überforderung entgegenwirken. Neurodoron ${ }^{\circledR}$ Tabletten und Calmedoron ${ }^{\circledR}$ Streukügelchen oder deren Mischung helfen bei stressbedingter Erschöpfung und Unruhe.
Neurodoron ${ }^{\circledR}$, eine arzneiliche Komposition aus potenziertem Gold, Kalium phosphoricum und Ferrum-Quarz, stärkt die Nerven bei Erschöpfung und harmonisiert das bei Stress gestörte Zusammenspiel der körperlichen, seelischen und geistigen Ebenen im Organismus. Dabei macht es nicht müde oder beeinträchtigt die Konzentration. Es sorgt für innere Ruhe, indem es bei nervöser Erschöpfung Angst- und Unruhezstände, Kopfschmerzen und depressive Verstimmung lindert. Die natürlichen Pflanzenauszüge aus Hopfen, Baldrian, Passionsblume und Hafer in Calmedoron ${ }^{\circledR}$ beruhigen bei Unruhe, Nervosität und Einschlafstörungen. Abends eingenommen fördert Calmedoron ${ }^{\circledR}$ das Einschlafen und unterstützt einen erholsamen und entspannten Schlaf.

Weitere Informationen bei

Weleda AG

www.weleda.de

\section{Mehr Sicherheit bei Multi- oder Langzeitmedikation durch Silymarin}

Medikationssicherheit ist ein wichtiger Aspekt bei der Behandlung von multimorbiden Patienten oder Patienten, bei denen Medikamente über einen längeren Zeitraum erforderlich sind. Denn bei einer Multi- oder Langzeitmedikation können unerwünschte Wechselwirkungen auftreten oder Nebenwirkungen verstärkt werden. So werden viele Medikamente in der Leber metabolisiert. Das Risiko einer medikamentös-toxischen Leberschädigung wird deutlich erhöht. Dieser sollte rechtzeitig präventiv begegnet werden, z.B. mit Legalon ${ }^{\circledR}$ (Silymarin). Eine Studie belegt, dass Silymarin zu einer deutlichen Verbesserung der Leberwerte führt [1]. Die nichtinterventionelle Studie wurde mit 190 Patienten durchgeführt, die mindestens 1 potenziell lebertoxisches, nicht absetzbares Medikament mit einer medianen Einnahmedauer von 2,8 Jahren einnahmen. Das Ziel der 4-monatigen prospektiven Studie war es, zu untersuchen, welchen Einfluss Legalon auf die Lebergesundheit und Lebensqualität der Patien- ten hat [1]. Bereits nach 2 Monaten war ein deutlicher Effekt zu beobachten. Nach weiteren 2 Monaten war eine signifikante Verbesserung hinsichtlich aller Leberwerte im Vergleich zum Studienbeginn zu verzeichnen (jeweils $p<0,001$ ). Zu Studienende lag der Anteil der Patienten, die einen ALT-Wert im Normbereich hatten, bei 40,0\%, bezüglich AST bei 47,9\%, GGT bei 33,2\%, alkalischer Phosphatase bei $67,4 \%$ und bezüglich Gesamtbilirubin bei 66,8\% [1]. Auch der Anteil der Patienten ohne Lebensqualitätseinschränkung stieg innerhalb des Untersuchungszeitraums signifikant an [1]. Zu Studienbeginn sowie nach 2 bzw. 4 Monaten lag der Anteil bei 10,0\%, 24,2\% bzw. 35,3\% [1].

\section{Literatur}

1 Gillessen A et al.: MMW-Fortschritte der Medizin; Originalien Nr IV/2014.

MEDA Pharma GmbH \& Co. KG / www.medapharma.de 


\section{News}

Forsch Komplementmed 23 | 6 | 16

DigiConPro

\section{Eine neue Dimension digitalisierter Substanzen}

Der komplementärmedizinisch arbeitende Arzt oder Therapeut kennt den Wert des Einsatzes von Stoffen oder Substanzen, ob nativ oder homöopathisch, zur Testung und Behandlung. Die elektromagnetische Abstrahlung jeder Substanz und jedes Stoffes (natürlich vorkommend oder künstlich reproduziert) ist wie ein Fingerabdruck - einzigartig.

Substanzen in digitalisierter Form anzubieten ist heute keine Neuheit mehr. Auf dem Markt werden meist Lösungen auf der Grundlage von Soundkarten angeboten, wie wir sie aus der Computerwelt kennen - also Systemen, die sich auf den Hörbereich beschränken, in einer Bandbreite von $20 \mathrm{~Hz}$ bis ca. $20000 \mathrm{~Hz}$. Zusätzlich liegen (durch die Elektronik unvermeidbare) Umgebungsgeräusche wie ein «Grauschleier» darüber und vernebeln damit ihre Klarheit.

Je breitbandiger und reiner die Aufnahmeund Wiedergabequalität, desto zielgerichteter und durchdringender ist die Anwendung und desto besser auch ihre Verträglichkeit, vor allem bei chronisch belasteten Fällen. Um den

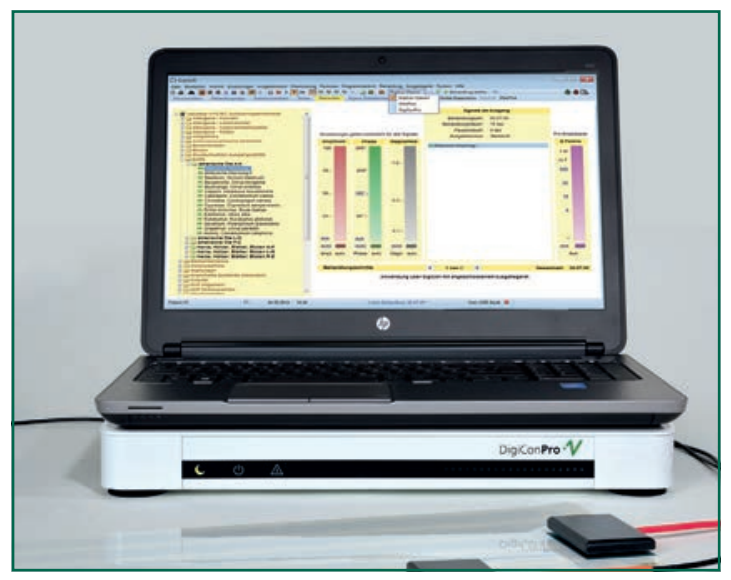
VITATEC Medizintechnik GmbH 0817792940

deutschland@vitatec.com www.vitatec.com und Bandbreite zur Verfügung stehen. hertz) ermöglicht. haft in hoher Qualität verfügbar. niert werden.
"Gesundheit aus dem Meer" 50. Medizinische Wochen Baden-Baden

Nutzwert zu steigern, sollte ein Fingerabdruck in einer erheblich höheren Qualität

Deshalb hat VITATEC nach Jahren der Beobachtung und Erfahrung DigiConPro entwickelt, das Substanzspektren in einer Bandbreite von 1 bis $2000000000 \mathrm{~Hz}$ (2 Giga-

Die bisher ca. 10700 erfassten Substanzen werden auch zukünftig erweitert. Durch die rein physikalische Testung und Anwendung sind die Möglichkeiten der Dosierung, Verfeinerung, Potenzierung usw. gegenüber einer nativen Substanz praktisch grenzenlos. Und wäre eine Originalsubstanz eines Tages zu schwach geworden oder gar nicht mehr erhältlich, bleibt sie mit diesem System dauer-

DigiConPro wird mit Hilfe einer ausgereiften Software über einen Laptop gesteuert. Die ausgewählten Substanzen (Einzelsubstanzen, Raffampullen u.v.m.) werden über eine speziell dafür entwickelte Elektrode auf den Körper appliziert. Das System kann zur Anregung oder Entlastung, zur Regulierung oder Ausleitung sowie zur Begleitung jeder anderen Maßnahme angewendet werden. Es ist zur alleinigen Nutzung geeignet oder kann mit jedem anderen System oder Konzept kombi-

\section{Excellence Research Award 2016 für Akupunkturforschung}

Für ihre wissenschaftliche Studie «Akupunktur-induzierte Veränderungen der Druckschmerzschwelle durch segmentale Hemmung - eine randomisierte, kontrollierte Studie» wurde ein Forscherteam um Petra Bäumler und PD Dominik Irnich mit dem renommierten «Excellence in Integrated Medicine Research Award 2016"* in der Kategorie Grundlagenforschung ausgezeichnet. Petra Bäumler ist Mitglied des Wissenschaftszentrums der DÄGfA und wissenschaftliche Mitarbeiterin der Interdisziplinären Schmerzambulanz am Campus Innenstadt der LMU München. Dominik Irnich ist Leiter der Interdisziplinären Schmerzambulanz am Campus Innenstadt der LMU München und 1. Vorsitzender der DÄGfA. Die Preisträger konnten in ihrer Arbeit erstmals die Bedeutung der spinalen Hemmung für die Akupunkturwirkung beschreiben [1]

\section{Literatur}

1 Baeumler PI et al.: PAIN 2015;156:2245-2255

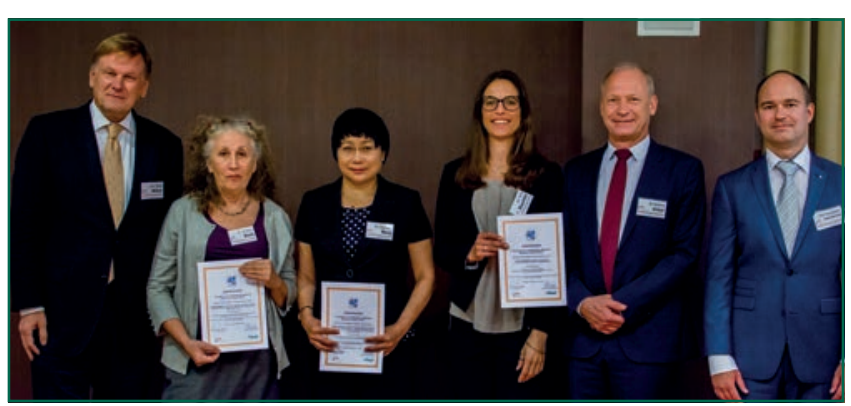

v.I.n.r.: Prof. S. N. Willich (Vorsitzender der Jury); die Preisträger C. Wade, Prof. C. Wang, P. Bäumler, PD D. Irnich und Dr. K. Cesnulevicius (Biologische Heilmittel Heel GmbH) * Der internationale Preis wird von der Biologische Heilmittel Heel GmbH gefördert.

DÄGfA Deutsche Ärztegesellschaft für Akupunktur e.V. / www.daegfa.de
Das waren in der Tat spannende Vorträge, die beim Symposium "Gesundheit aus dem Meer» auf der 50. Medizinischen Woche in Baden-Baden präsentiert wurden.

Jörg Ullmann berichtete über «Chlorella: neue Ansätze zur Prävention und Therapie zivilisationsbedingter Stoffwechselstörungen» und ging hier vor allem auf das Thema Vitamin B12 aus der Mikroalge Chlorella ein sowie auf die über Fettsäuren der Chlorella aktivierte PPAR-vermittelte Genexpression als Zielmolekül für bestimmte Arzneimittel, um Krankheiten wie Hyperglykämie, Diabetes und Obesitas zu bekämpfen.

Dr. Bettina Hees berichtete über «Astaxanthin beim Computer Vision Syndrom». Astaxanthin ist ein natürliches Antioxidans, gewonnen aus der Mikroalge Haematococcus pluvialis, das aufgrund seiner besonderen Molekularstruktur in der Lage ist, die BlutRetina-Schranke zu überwinden und direkt in der Netzhaut für einen antioxidativen und entzündungshemmenden Schutz des Auges zu sorgen.

Dominik Hoffmann sprach zum Thema "Algen und Darmgesundheit» und betonte die für ein gesundes Darmmilieu wichtige Symbiose zwischen präbiotisch wirkenden Algen und probiotischen Bakterien.

Roquette Klötze GmbH \& Co. KG

Jörg Ullmann

www.algomed.de

Marine Therapy Solutions $\mathrm{GmbH}$

Dr. Bettina Hees

www.marine-therapy.com

HLH BioPharma Vertriebs GmbH

Dominik Hoffmann

www.algomed.de 